\title{
The IMaX polarimeter for the solar telescope SUNRISE of the NASA long duration balloon program
}

\author{
A. Alvarez-Herrero ${ }^{1}$, V. Martínez-Pillet ${ }^{2}$, J. C. del Toro Iniesta ${ }^{3}$, V. Domingo ${ }^{4}$ and the IMaX team. \\ ${ }^{1}$ Insitituto Nacional de Técnica Aeroespacial-INTA, Torrejón de Ardoz, 28850 Madrid, Spain. \\ ${ }^{2}$ Instituto de Astrofísica de Canarias-IAC. La Laguna, 38200 Tenerife, Spain. \\ ${ }^{3}$ Instituto de Astrofísica de Andalucía-IAA (CSIC). P.O. Box 3004, 18080 Granada, Spain. \\ ${ }^{4}$ Grupo de Astronomía y Ciencias del Espacio-GACE (UV). Paterna, 46980 Valencia, Spain.
}

\begin{abstract}
On June 8th 2009 the SUNRISE mission was successfully launched. This mission consisted of a $1 \mathrm{~m}$ aperture solar telescope on board of a stratospheric balloon within the Long Duration Balloon NASA program. The flight followed the foreseen circumpolar trajectory over the Artic and the duration was 5 days and 17 hours. One of the two postfocal instruments onboard was IMaX, the Imaging Magnetograph eXperiment. This instrument is a solar magnetograph which is a diffraction limited imager capable to resolve $100 \mathrm{~km}$ on the solar surface, and simultaneously a high sensitivity polarimeter $\left(<10^{-3}\right)$ and a high resolution spectrograph (bandwidth $<70 \mathrm{~m} \AA$ ). The magnetic vectorial map can be extracted thanks to the well-know Zeeman effect, which takes place in the solar atoms, allowing to relate polarization and spectral measurements to magnetic fields. The technological challenge of the IMaX development has a special relevance due to the utilization of innovative technologies in the Aeroespacial field and it is an important precedent for future space missions such as Solar Orbiter from ESA. Among these novel technologies the utilization of Liquid Crystal Variable Retarders (LCVRs) as polarization modulators and a $\mathrm{LiNbO}_{3}$ etalon as tunable spectral filter are remarkable. Currently the data obtained is being analyzed and the preliminary results show unprecedented information about the solar dynamics.
\end{abstract}

\section{Introduction}

The goal of SUNRISE mission is the study of the solar magnetic fields analysing the features with temporal dependency of the magneto-convective patterns, as well as the interaction at small scale between the convective fluxes and the magnetic field. In conclusion, the objective is to study the magnetic field of the Sun with high spatial resolution, obtaining relevant keys to understand the dynamic of our star and answer questions as the origin of the high temperature in the solar corona (around $2.10^{6} \mathrm{~K}$ against $5600 \mathrm{~K}$ in the inner photosphere) or the connexion with the so called Space Weather, which has a fundamental importance for our daily life.

The SUNRISE mission consists of a stratospheric balloon with a $1 \mathrm{~m}$ aperture solar telescope onboard. It was successfully launched on June 8th 2009 within the Long Duration Balloon NASA program. The flight followed the foreseen circumpolar trajectory over the Artic having a duration of 137 hours.

The Imaging Magnetograph eXperiment, IMaX, one of the two SUNRISE post-focal instruments, is a solar magnetograph. It is a diffraction limited imager in order to resolve $100 \mathrm{~km}$ on the solar surface, but additionally is a high sensitivity polarimeter $\left(<10^{-3}\right)$ and a high resolution spectrograph $(\Delta \lambda<70 \mathrm{~m} \AA)$. To fulfil these requirements, IMaX utilizes two innovative technologies applied to an aerospace instrument: Liquid Cristal Variable Retarders (LCVRs) and a tuneable $\mathrm{LiNbO}_{3}$ etalon.

IMaX has been developed by a Spanish consortium coordinated by the Instituto de Astrofísica de Canarias (IAA) formed by the Instituto Nacional de Técnica Aerospacial (INTA), the Instituto de Astrofísica de Andalucía (IAA-CSIC) and the Grupo de Astronomía y Ciencias del Espacio of the Universidad de Valencia (GACE-UV). The Instituto Ignacio da Riva of the Universidad Politécnica de Madrid (IDR-UPM) also participated in the SUNRISE mission carrying out tasks at system level. The duration of the IMaX development was seven years and a half, eighteen months dedicated to the Assembly, Integration and Verification phase (AIV phase). The SUNRISE mission was leaded by the MaxPlank-Insitut für Sonnensystemforsschung (MPS, Germany) with the contributions of KIS (Germany), HAO-NCAR (USA) and Lockheed-Martin LMSAL (USA). 
Fig. 1. SUNRISE in the launching pad during the balloon inflating phase, some minutes before the lift-off. In the front of the picture, the gondola held by an auxiliary crane can be seen. The telescope is in the center between the solar panels. Over the telescope, the Post-Focus Instrumentation (PFI) is allocated with IMaX and SUFI.

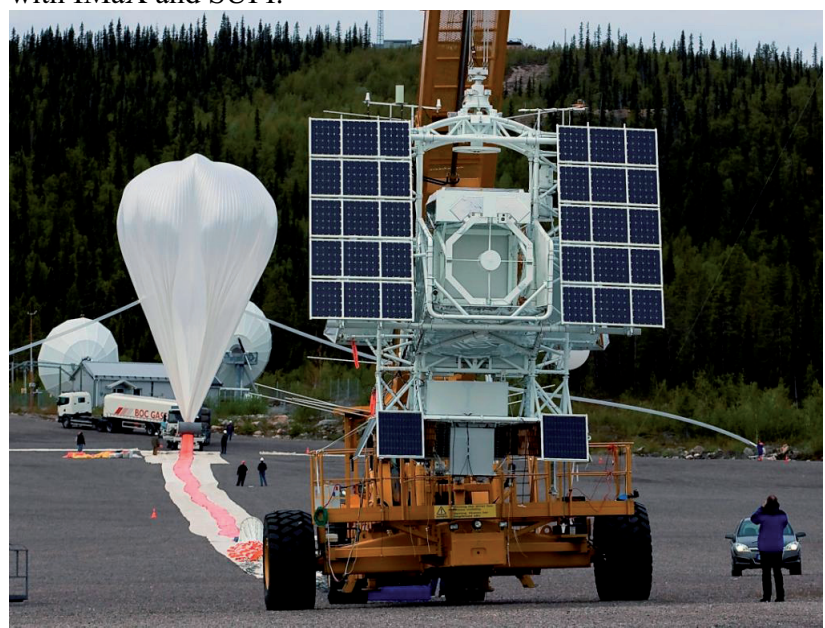

\section{Scientific goals and instrument requirements}

The main scientific goal of SUNRISE and the implications in the instruments requirements of the payload are the following:

- Resolve small magnetic elements with sizes lower than $100 \mathrm{~km}$ in the solar surface: that implies to develop a telescope of $1 \mathrm{~m}$ aperture diffraction limited and to place it outside of the atmosphere to avoid perturbations and to be able to detect the required UV wavelengths. Additionally, a high precision pointing system and image stabilization system are required. Since $100 \mathrm{~km}$ in the Sun surface means an angular resolution of $0.14 \mathrm{arcsec}$, the pointing and stabilization system shall be better than 0.005 arcsec.

- Resolve temporal evolutions lower than 5 seconds and cover hours/days: it implies high acquisition cadence and uninterrupted observations.

- Measure the 3D distribution of the magnetic vector, velocity and temperature: the suitable technique to obtain these data is polarization sensitive spectroscopy in the photospheric and chromospheric spectral lines.

- High cadence imaging of different solar layers: therefore, it is necessary the development of imagers with spectral filters for the visible and the UV.

\section{SUNRISE mission}

As it was describe previously, SUNRISE is an optical telescope onboard of a stratospheric balloon which has planned a succession of long duration flights in circumpolar trajectories with uninterrupted periods of observation to obtain temporal sequences of diffraction limited images. It has two postfocal instruments: SUFI, the $\underline{\boldsymbol{S U}}$ NRISE Filter Imager and IMaX, the Imaging Magnetograph experiment. SUFI is a UV imager and IMaX a visible spectroscopic imager polarimeter. In the instrumental platform is also placed the Correlation Tracker and $\underline{\boldsymbol{W}}$ avefront $\underline{\boldsymbol{S}}$ ensor (CWS) which is in charge of the real-time image analysis to provide the feedback to the image stabilization mechanisms.

In the gondola are included the telescope azimuth and elevation stabilization systems (accuracy $\sim 10-40$ arcsec), the power subsystems (solar panels and batteries) as well as the telemetry and telecommand subsystem. These elements were verified in a test flight carried out in New Mexico (USA) in October 2007.

SUNRISE flew in the Artic from the ESRANGE base (Kiruna, Sweden) within the Long Duration Program of NASA. The scientific payload weighed around $1920 \mathrm{~kg}$ and the total mass was 6.09 tons. The flight altitude typically was around $35-40 \mathrm{~km}$ and, to achieve this, a balloon of approximately $130 \mathrm{~m}$ of diameter with a volume of $835.000 \mathrm{~m}^{3}$ was utilized. The pressure at the flight altitude was $3-7 \mathrm{mbar}$ with the resulting difficulties in the high voltage handling (increase the risk of arc discharges), the heat transport mechanisms (only conduction and radiation, no convection) and the change of the focal plane position among others.

\section{IMaX instrument description}

The IMaX working is based on deducing the magnetic field from the polarimetric and spectroscopic measurements of some specific absorption spectral lines sensitive to the Zeeman effect. The magnetic field splits the spectral lines corresponding to the levels of the atoms in Sun being each of them polarized. That occurs in the case of the magnetic vector component in the line of sight and the transversal component. In the practice, the Stokes vector of every pixel in the image is measured for several selected wavelengths, scanning in this way the spectral line of interest.

Fig. 2. Sketch of the Optical Unit of IMaX.

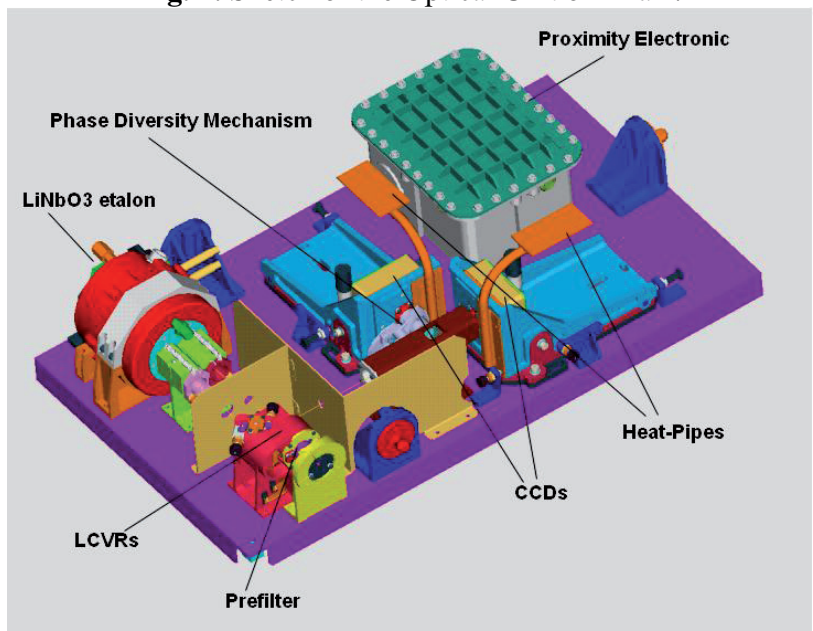

Table 1 shows the specifications imposed to the optics system by the scientific requirements and the achieved values in the final design for laboratory conditions (LabC). Also the expected values during flight considering the thermal conditions foreseen by the instrument thermal design are presented. 
Table 1. Main optical parameters. Comparison of the specified values, the obtained values in the final design at laboratory environmental conditions (LabC) and the expected values during operation.

\begin{tabular}{|c|c|c|c|}
\hline PARAMETER & SPECS & $\begin{array}{l}\text { VALUE at } \\
\text { LabC }\end{array}$ & $\begin{array}{l}\text { VALUE at } \\
\text { operation }\end{array}$ \\
\hline Spectral Range & $\begin{array}{l}525.02 \\
\pm 0.2 \mathrm{~nm}\end{array}$ & $\begin{array}{c}525.02 \\
\pm 0.2 \mathrm{~nm}\end{array}$ & $\begin{array}{c}525.02 \\
\pm 0.2 \mathrm{~nm}\end{array}$ \\
\hline FOV $(\mathrm{XxY})$ & $\begin{array}{l}>50 \times 50 \\
\operatorname{arc~sec}\end{array}$ & $\begin{array}{l}>50 \times 50 \\
\operatorname{arc~sec}\end{array}$ & $\begin{array}{l}>50 \times 50 \\
\operatorname{arc~sec}\end{array}$ \\
\hline Distortion & -- & $-0.06 \%$ & $0.012 \%$ \\
\hline $\begin{array}{l}\mathrm{F} / \mathrm{N} \text { in the } \\
\text { image plane }\end{array}$ & 45 & 44.99 & 45.01 \\
\hline $\begin{array}{c}\text { Image scale } \\
\text { (arcsec/pixel) }\end{array}$ & 0.055 & 0.055 & 0.055 \\
\hline $\begin{array}{l}\text { Incidence } \\
\text { angle of the } \\
\text { collimated } \\
\text { beam }\end{array}$ & $<0.58^{\circ}$ & $0.44^{\circ}$ & $0.44^{\circ}$ \\
\hline $\begin{array}{c}\text { WFE Phase } \\
\text { Diversity }\end{array}$ & $\begin{array}{c}0.25 \lambda \mathrm{rms} \\
( \pm 12 \%)\end{array}$ & $0.25 \lambda \mathrm{rms}$ & $0.28 \lambda \mathrm{rms}$ \\
\hline $\begin{array}{c}\text { Strehl ratio } \\
\text { (nominal / with } \\
\text { tolerances) }\end{array}$ & $0.95 / 0.8$ & $0.998 / 0.85 \mathrm{~s}$ & $0.998 / 0.83$ \\
\hline $\begin{array}{c}\text { Telecentrity in } \\
\text { the image } \\
\text { plane }\end{array}$ & $\begin{array}{l}\text { Telecentric } \\
\pm 60 \operatorname{arcsec}\end{array}$ & $\begin{array}{l}\text { Telecentric } \\
\pm 22 \operatorname{arcsec}\end{array}$ & $\begin{array}{l}\text { Telecentric } \\
\pm 35 \text { arcsec }\end{array}$ \\
\hline $\begin{array}{l}\text { Straylight and } \\
\text { ghost images }\end{array}$ & $<1 \%$ PSF & $\begin{array}{c}<1 \% \text { PSF } \\
\text { Tilting etalon } \\
0.36^{\circ}\end{array}$ & $\begin{array}{c}\text { Not } \\
\text { evaluated }\end{array}$ \\
\hline
\end{tabular}

The nominal final design [1] was performed using CODE $\mathrm{V}$ and fulfilled the requirements including the margins to take into account the manufacturing and system alignment tolerances. In the design phase simulations to know the effect in the optical performaces of the foreseen conditions by the thermal analytical models during the flight were performed. Besides, the required paints and the baffles to control the straylight were determined using simulations with ASAP software (Breault Research Organization). These studies also concluded the necessary actions to avoid the apparition of ghost images. Additionally, from the straylight analysis the required cleanness level of the optical surfaces was specified as better that 200 (see MIL-STD-1246C and FED-STD-209E). This last conclusion implied a specific cleanness control during the AIV phase.

The design includes a parallel plate which can be inserted using a mechanism in order to produce a known defocus. Through the analysis of couples of images focused/unfocused is possible to recover the distorted images by unknown causes using the Phase Diversity Method [2].

The polarization state of the light is analyzed for every pixel of the image determining the Stokes vector in order to deduce the magnetic vector for every point in the image and, afterwards, to obtain a map of the magnetic field. The polarization analysis is carried out using two Liquid Crystal Variable Retarders (LCVRs) and a polarizing beam splitter. Applying low voltages $(<14 \mathrm{~V})$, four known polarization modulations were introduced and then, the four components of the Stokes vector were extracted [3]. The utilization of LCVRs in space instrumentation is very promising because it avoids rotary mechanisms (i.e.: rotary polarizers or retarders schemes) or high voltages (i.e.: photo-elastic modulators). Nevertheless, since LCVRs is a novel technology applied to an instrument with space operational conditions a very exhaustive test plan was carried out to characterize and to verify their suitable performance during the flight [4]. The LCVRs were developed by the Spanish company TECDIS Iberica, currently called Visual Display S.L..

Fig. 3. Calculated demodulation matrix from the modulation matrix of the LCVRs, at $35^{\circ} \mathrm{C}$ and and vacuum $\left(9 \cdot 10^{-2}\right.$ mbar $)$

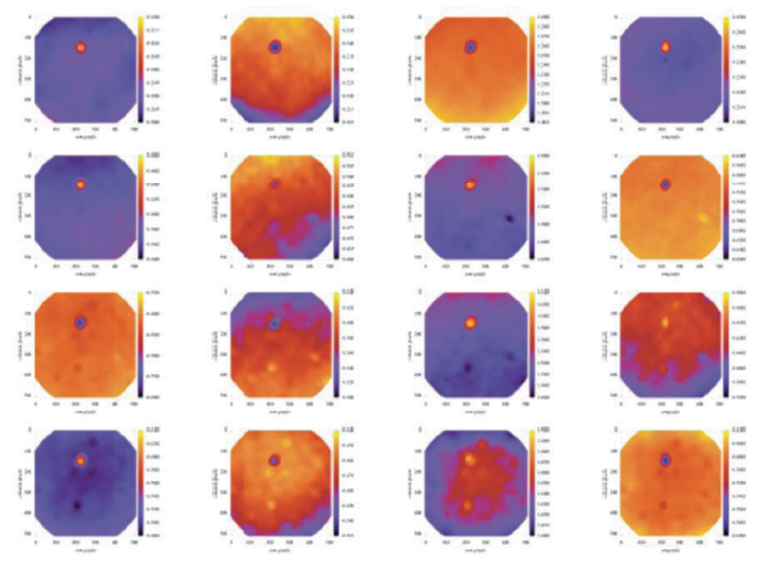

The obtained polarization modulation efficiencies [5] were close to the theoretical maximum as it can see in Table 2.

Table 2. Modulation efficiencies from the polarization modulation matrix at $35^{\circ} \mathrm{C}$ at room pressure and vacuum $\left(9 \cdot 10^{-2}\right.$ mbar $)$

\begin{tabular}{|c|c|c|c|}
\hline $\begin{array}{c}\text { Modulation } \\
\text { Efficiencies }\end{array}$ & $\begin{array}{c}\text { Theoretical } \\
\text { maximum }\end{array}$ & $\begin{array}{c}\mathrm{T}=35.0^{\circ} \mathrm{C} \pm 0.1^{\circ} \mathrm{C} \\
\mathrm{P}=\text { Ambient }\end{array}$ & $\begin{array}{c}\mathrm{T}=35.0^{\circ} \mathrm{C} \pm 0.1^{\circ} \mathrm{C} \\
\mathrm{P}=9 \cdot 10^{-2} \text { mbar }\end{array}$ \\
\hline$\varepsilon_{1}$ & 1 & $\begin{array}{c}0.991 \\
\pm 0.003\end{array}$ & $\begin{array}{c}0.987 \\
\pm 0.003\end{array}$ \\
\hline$\varepsilon_{2}$ & 0.577 & $\begin{array}{c}0.570 \\
\pm 0.005\end{array}$ & $\begin{array}{c}0.572 \\
\pm 0.004\end{array}$ \\
\hline \multirow{2}{*}{$\varepsilon_{3}$} & 0.577 & $\begin{array}{c}0.580 \\
\pm 0.006\end{array}$ & $\begin{array}{c}0.568 \\
\pm 0.006\end{array}$ \\
\hline \multirow{2}{*}{$\varepsilon_{4}$} & 0.577 & $\begin{array}{c}0.531 \\
\pm 0.007\end{array}$ & $\begin{array}{c}0.529 \\
\pm 0.007\end{array}$ \\
\hline
\end{tabular}

To obtain solar magnetographs requires high resolution imaging and high sensitive polarimetric measurements, but also tuning simultaneously wavelengths with high resolution $(<70 \mathrm{~m} \AA)$ in order to scan the absorption spectral line under study (Fe I 5250.2 $\AA$ ). IMaX uses a $\mathrm{LiNbO}_{3}$ etalon developed by APO/CSIRO (Australian Precision Optics, CSIRO) for spectral tuning applying a high voltage which induces the Pockels effect and, therefore, avoiding mechanical tuning mechanisms. The etalon was placed in double pass configuration in order to obtain the required bandwidth avoiding including a second etalon with the associated problems of synchronism and duplication of high voltage supplies [6]. 
To eliminate the unwanted transmitted orders by the etalon a prefilter $1 \AA$ width (FWHM) was introduced.

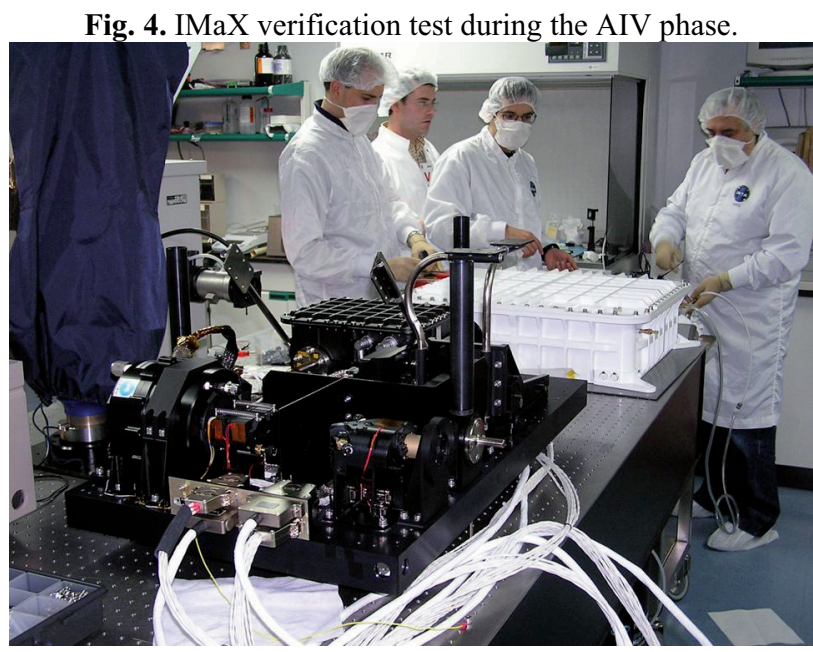

After finalising the IMaX design which took five years and a half, the manufacturing and AIV phases were undertaken. The IMaX AIV phase took 12 months and many activities were carried out to guarantee the proper working of the instrument in operational conditions. Specially, the performed environmental tests are remarkable which included thermal cycles to verify the IMaX optical performances measuring in situ parameters as the MFT, focus, etc., as well as checking the functionality of the etalon tuning and the LCVRs polarization modulation. In December 2008 IMaX was ready to be delivered to the MPS (Germany) and to proceed with its integration in the SUNRISE instrument platform. This task was performed during the first months of 2009. Afterwards, all the SUNRISE components and subsystems were transported to the ESRANGE base (Kiruna, Sweeden) in the Artic were the final integration and the flight preparation was carried out.

\section{Preliminary results}

The telemetry and telecomand line during the SUNRISE flight had a low bandwidth for the operation of the system. The scientific data were recorded in the hard disks onboard and recovered later. Figure 5 shows the first official SUNRISE image which was obtained by IMaX. The resolution and extension was reduced to be transmitted by the telemetry line. However its high quality and excellent contrast can be appreciated. Nowadays, the scientific team is processing and analyzing the obtained data, although some conclusions have been already extracted:

-The IMaX quality data during the long observation time obtained has never achieved before.

-The image contrast is around 8.5\%. After applying the Phase Diversity method the contrast achieves 13\%-15\% close to the theoretical maximum.

-After processing the data with the Phase Diversity method, the angular resolution is better 0.18 arcsec and probably achieves the diffraction limit $(0.14 \operatorname{arcsec})$.
The team is preparing numerous publications to spread these important results. For all these reasons the first flight of SUNRISE is already considered a great successful by the international scientific community.

Fig. 5. SUNRISE first image. Granulation at disk center as seen in one of the modulation states of the IMaX instrument. Preliminary processed thumbnail with a $4 \times 4$ binning. $256 x 256$ pixels $(0.22 \% / p x)$. Exposure time: 1.5 s. $\lambda=525.04 \mathrm{~nm}$. FoV: 56".

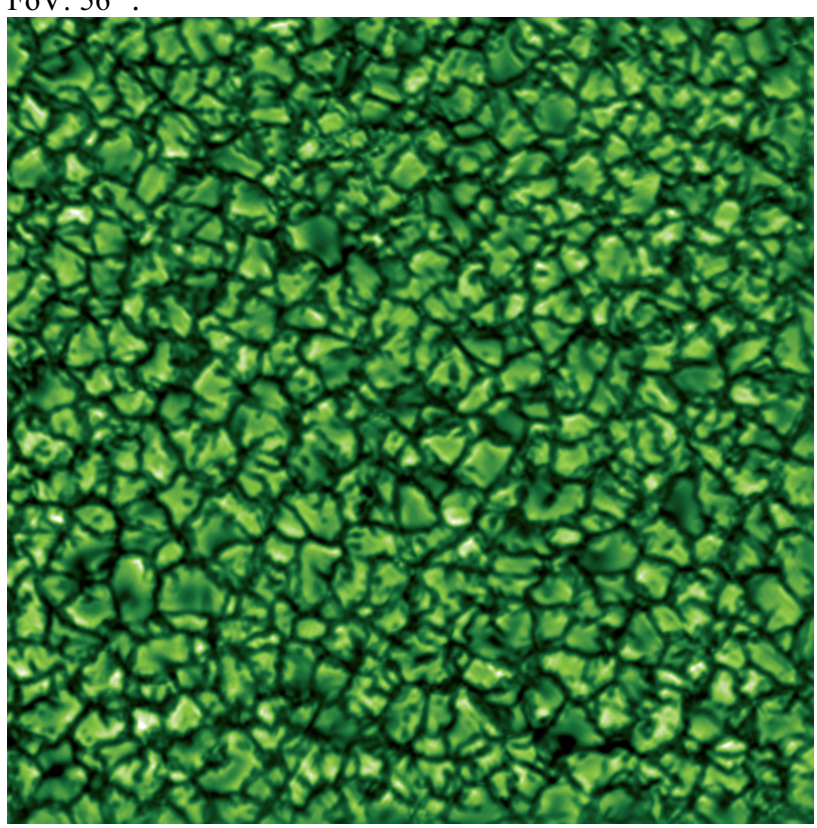

\section{Conclusions}

The preliminary results of SUNRISE confirm the success of the first flight of the mission. In addition to the data analysis and the preparation of a possible second flight, the international consortium has been selected and is currently involved in the development of the SO/PHI instrument (Polarimetric and Heliosesmic Imager) for the Solar Orbiter space mission of the European Space Agency (ESA). It includes the activity to validate the utilization of LCVRs in the mission. IMaX can be considered as the precursor of SO/PHI since its baseline include the novel technologies implemented: the LCVRs as polarization modulator and the $\mathrm{LiNbO}_{3}$ etalon.

\section{Acknowledges}

The scientific and technologic team wants to thanks for its supporting to the Ministerio de Ciencia en Innovacion of Spain and for the successive awarded funding for the development and scientific exploitation of IMaX. Specifically this work has been funded with the project ESP-2006-13030 "Integration and flight of SUNRISE/IMaX. Conceptual phase of Solar Orbiter/VIM".

\section{References}

1. A. Alvarez-Herrero et al., SPIE Astronomical Telescopes and Instrumentation. Space Telescopes 
and Instrumentation I: Optical, Infrared and Millimetre. 6265, (2006).

2. R. G. Paxman, T. J. Schulz and J.R. Fienup, J. Opt.Soc. Am. A 9, 1072 (1992).

3. N. Uribe-Patarroyo, A. Alvarez-Herrero, R. L. Heredero, J. C. del Toro Iniesta, A. C. López Jiménez, V. Domingo, J. L. Gasent, L. Jochum, V. Martínez-Pillet and the IMaX Team, , Phys. Stat. Sol. C 5, 1041 (2008).

4. R. L. Heredero, N. Uribe-Patarroyo, T. Belenguer, G. Ramos, A. Sánchez, M. Reina, V. Martínez-Pillet and A. Alvarez-Herrero, Appl. Opt. 46, 689 (2007).

5. J. C. del Toro Iniesta, M. Collados, Appl. Opt. 39, 1637 (2000).

6. A. Alvarez-Herrero et al. SPIE Astronomical Telescopes and Instrumentation. Space Telescopes and Instrumentation I: Optical, Infrared and Millimetre, 6265 (2006). 